\title{
REVIEW
}

\section{Neurological rehabilitation: from mechanisms to management}

\author{
Alan J Thompson
}

This review is the content of the Inaugural Lecture of the Garfield Weston Chair of Clinical Neurology and Neurorehabilitation given by Professor A J Thompson at the Institute of Neurology, UCL, 10 July 1999

Department of Clinical Neurology, Institute of Neurology, UCL, Queen Square, London WC1N 3BG, UK A J Thompson

A.Thompson@ion.ucl.ac.uk

Received 14 April 2000 and in revised form

20 June 2000

Accepted 6 July 2000
The main messages contained within this review are firstly that any advance in the management of patients with neurological disability must come from improved understanding of the mechanisms underlying that disability and secondly that the only way to demonstrate real benefit is by a process of evaluation which must incorporate appropriate measuring tools. In other words, to emphasise the crucial role of the basic sciences (be it cellular biology, immunology, physiology, or imaging) in elucidating the mechanisms underlying disability and similarly to underline the importance of the scientific principles which underpin the outcome measures used to evaluate disease management.

The three great challenges currently facing the field of neurological rehabilitation are firstly to elucidate these mechanisms, secondly to ensure that this knowledge transfers speedily to the clinical arena, and thirdly to identify scientifically sound outcome measures with which to evaluate clinical intervention.

There have been several recent advances in our understanding of the mechanisms underlying disability in neurological disorders, and multiple sclerosis provides an excellent example. This is one of the main causes of neurological disability in young adults and recent work in this area has shown the value of scientific investigation which is driven by addressing important clinical questions. There have also been encouraging advances in measuring outcome in the field of neurological rehabilitation and these will be discussed with particular reference to their application to the evaluation of therapy and rehabilitation.

Multiple sclerosis is a complex neurological disorder perhaps best exemplified by its diverse patterns of disease activity, incorporating relapses and progression. ${ }^{1}$ The development of MRI has provided an invaluable diagnostic tool which may also be used to monitor and predict disease activity ${ }^{2}$ and evaluate therapeutic interventions. ${ }^{3}$ However, once the initial flush of enthusiasm passed, it became clear that there was a major discrepancy between the extent of the abnormalities seen on brain MRI and the impact the disease was having on the patient. ${ }^{4}$ This was particularly obvious in patients with primary progressive multiple sclerosis-who have the worst clinical prognosis. In the first serial study of this group in the late 1980s it was shown that, despite the fact that patients in this subgroup were getting progressively worse they showed very little new activity on MRI and even less inflammation. ${ }^{5}$ This discrepancy encouraged investigators to consider other reasons for their disability and stimulated the development of faster and more accurate imaging of the spinal cord-an area known to be both a common target and a major source of disability in multiple sclerosis. ${ }^{6}$ The improved imaging certainly confirmed these viewslesions were seen in most patients but, again, failed to explain their level of disability. These studies did, however, produce one interesting observation which has been subsequently exploited to the full-patients in the progressive phase of the condition were found to have marked tissue loss and the degree of cord atrophy correlated well with disability. ${ }^{7}$ Refinement of this measurement technique and relocalisation to a higher and more anatomically consistent level (C2) provided the scientific properties necessary for serial measurement which in turn demonstrated measurable change within as short a period as 1 year. ${ }^{8}$

This observation led to studies of atrophy in other areas, led by work from Losseff who developed a technique capable of measuring a defined volume of the brain at the periventricular level. ${ }^{10}$ Although this was only a partial measure of brain volume, it focused on the area of greatest potential change and was therefore sensitive to change over time and, perhaps more importantly, demonstrated an encouraging correlation with disability. Subsequently, a range of new techniques have been developed, confirming the initial observations and demonstrating that changes are detectable over a relatively short period of time, may be seen early in the disease course, ${ }^{11}{ }^{12}$ and have the potential to be useful outcomes in therapeutic trials. ${ }^{13}$

What do these observations mean pathologically and clinically? Atrophy could be described as an end stage process which may result from loss of various tissue components including myelin and axons. Clinically it could be the cause of the irreversible deficit seen in multiple sclerosis although that is likely to depend on the severity of the axonal loss and its precise location. There has been renewed interest in axonal loss in multiple sclerosis as a result of recent publications from Ferguson et $a l^{14}$ and Trapp et $a l,{ }^{15}$ reminding us of what has 
been recurrently described and forgotten since the time of Charcot. ${ }^{16}$

What is perhaps more important is to try and tease out the mechanisms behind the development of atrophy and its likely clinical correlate-irreversible disability. To do this, more pathologically specific imaging techniques are required, applied not just to the lesions but also to the normal appearing white matter, which has been shown repeatedly to be far from normal. ${ }^{17}$ These techniques, which include magnetisation transfer imaging and the evaluation of hypointense lesions or black holes, provide information on tissue integrity, correlate better with disability, and in the case of magnetisation transfer imaging, demonstrate abnormalities in the normal appearing white matter before the appearance of lesions. ${ }^{18}$ More recently, the potential of diffusion tensor imaging has been investigated. This form of imaging is based on the continuous random motion of molecules in fluid systems and the effect barriers such as white matter tracts have on that motion (a property termed anisotropy) and therefore provides information about the integrity of structures and their direction. This "directionality" can be used to identify fibre tracts-including the optic tract, corpus callosum, and corticospinal tract. If applied to an acute lesion, the disruption of tissue integrity causes a loss of fractional anisotropy in the lesion. Studies in multiple sclerosis have shown abnormalities in the normal appearing white matter and within lesions, most markedly in black holes and acute lesions. ${ }^{19}$ MR spectroscopy - and in particular its ability to measure concentrations of $\mathrm{N}$-acetyl aspartate (NAA) - is the most specific technique for identifying axonal dysfunction. A strong correlation between reduced NAA in the cerebellum and cerebellar ataxia has been demonstrated. ${ }^{20}$ Abnormalities in the normal appearing white matter have also been demonstrated, which may precede the development of new lesions. ${ }^{21} 22$

In summary, these techniques:

- Confirmed the heterogeneity of lesions seen on conventional imaging

- Showed a better (although still limited) correlation with disability

- Demonstrated varying degrees of abnormality in normal appearing white matter

- Suggested that more marked changes in normal appearing white matter may occur before the appearance of new lesions.

Powerful though MRI may be, it does not provide unequivocal pathological evidence of the underlying changes which cause disability. For this, it needs to be used in careful pathological studies such as that recently completed by Polman and Barkhof's group in Amsterdam. Their elegant and illuminating study of the histopathology of multiple sclerosis lesions and normal appearing white matter, focusing on axonal loss, demonstrated a very high correlation between magnetisation transfer ratio, black holes, and axonal loss. ${ }^{23}$

In summary, these studies indicate that axonal loss is important in the development of disability. A key issue is the relation between the main pathological processes: inflammation, demyelination, and axonal loss, the elucidation of which requires the serial application of MR measures which reflect each of the pathological processes to cohorts of patients representative of each of the clinical subpopulations.

When considering the relation between pathology and its effect on the patient, the other part of the equation is the way in which disease impact is measured clinically. One of the great limitations of previous and current studies in multiple sclerosis is that they have had to rely on a single scale-Kurtzke's expanded disability status scale (EDSS) ${ }^{24} \mathrm{~A}$ key factor is that its development began almost 45 years ago, in 1955 and this is relevant if we consider the development of measurementthe basic scientific principles of which were established by mathematical psychologists in the mid-1800s. A major breakthrough was the demonstration that these psychophysical scaling methods could be used to accurately measure human attributes. This resulted in a series of standardised tests used in areas such as the assessment of educational achievement. ${ }^{25}$ Of particular relevance is the fact that this expertise did not effectively transfer to the field of health care evaluation until the 1970s, and even since then, clinicians have been rather slow to refer to these conceptual and methodological developments, possibly because the literature is not easily accessible (and when it is found, it may border on the incomprehensible). These psychometric principles are fundamental to the development of scientifically sound measurement - that is, measures that are reliable, valid, and responsive - which in simple terms means that a scale must be free from random error, measure what it purports to measure, and be sensitive to change. ${ }^{26}$ Of course, these essential qualities are interdependent. Clinical scales must also be appropriate to the population under study, which should reflect the entire range of the measure with minimal floor and ceiling effects. ${ }^{27}$ It is therefore logical that the choice of outcome measure should inform and influence trial design including patient selection, duration of study, and the numbers of patients required to provide sufficient power. Unfortunately, all too often the outcome measure is more of an afterthought.

The second major measurement issue, in which there has been considerable development, is the impact or consequences of disease on the individual patient. This is particularly relevant in the field of neurological rehabilitation. The World Health Organisation attempted to clarify this impact by producing the International Classification of Impairment, Disability, and Handicap (ICIDH) - each component of which may be defined, distinguished, and measured. ${ }^{28}$ Impairment is essentially the structural abnormality (for example, leg weakness) whereas disability reflects limitation in carrying out tasks (for example, walking) and handicap, restriction on participation in society (for example, using transport). Commonly used generic instruments which usefully illustrate the areas 
include the Barthel Index ${ }^{29}$ and Functional Independence Measure ${ }^{30}$ for disability, and the London Handicap Scale. ${ }^{31}$ Within this development there is a growing move towards incorporating the patient's perspective of disease impact (quite different from the physician's perspective).$^{32-34}$ This philosophy is contained within the concept of health related Quality of Life, a good example of which is the Medical Outcome Study 36-item short form health survey $(\mathrm{SF}-36) .^{35}$

So why are these developments in psychometrics so important and potentially useful? They allow us to do various things: (1) evaluate existing scales; (2) compare scales; (3) develop new scales; (4) evaluate the underlying constructs on which scales are based.

As an illustration, and returning to the area of multiple sclerosis, it is useful to look at the EDSS, which was developed before the application of psychometrics and is based on clinical intuition and expertise. It mixes impairment and disability and is mobility biased. However, on considering its scientific properties, major limitations regarding its reliability, and particularly its responsiveness, are apparent. ${ }^{36}$ The EDSS is considerably less responsive than generic measures of disability such as the Barthel Index and Functional Independence Measure in patients with multiple sclerosis undergoing rehabilitation. ${ }^{37}$ This may play some part in the obvious difficulty evaluating therapeutic agents in recent trials in multiple sclerosis.

Hobart et al have used psychometric techniques to compare the Functional Independence Measure and Barthel Index, and also to evaluate the second. The Barthel Index was developed in the 1950 s but it was thought that the range of disabilities it addressed was too limited and therefore the broader, bigger Functional Independence Measure was developed. Perhaps surprisingly, this increase in size provided no additional benefit from the measurement perspective. ${ }^{38}$ On the same theme-that is, that bigger is not necessarily better, Hobart has carried out a detailed evaluation of the Barthel Index itself on a cohort of 763 patients. In this study, an item analysis was carried out on 382 patients, which reduced the Barthel Index from 10 items to five. This five item measure was then evaluated in the other 381 patients and was shown to be as reliable, valid, and responsive as the larger measureand easier to use in clinical practice. ${ }^{39}$

Finally, and perhaps most importantly, this methodology allows the development of new scales in a scientifically sound fashion. There are five well defined steps in this process, the most fundamental being data collection, which is done from various sources, the most important of which is a series of in depth patient interviews. From this large interview based data set, together with other sources of information, all elements are extracted and a comprehensive (and usually large) measure is constructed. This is then field tested, analysed, and subsequently reduced. The measure then needs to be rigorously tested to ensure that it contains all the scientific properties necessary for a reliable, valid, and responsive measure. Within the Neurological Outcome Measure Unit at the Institute of Neurology a disease impact measure for multiple sclerosis is currently being developed..$^{40} \mathrm{It}$ is based on in depth patient interviews which produced 3750 items on a wide range of clinical issues. This resulted in a measure containing 141 items which after the first field test could be reduced to 29 items without losing information. This measure is currently being field tested for the second time.

It is only with the availability of such measures that we will be able to adequately evaluate interventions in diseases such as stroke and multiple sclerosis in a way which is relevant to the patient. This is certainly the case for drug trials but perhaps even more so for studies of neurological rehabilitation.

And what area could be more in need of evaluation? Rehabilitation is an immensely time consuming, labour intensive, and costly process involving many disciplines, many meetings and often lengthy inpatient admissions. However, there are major difficulties in attempting its evaluation, not least of which is its all encompassing remit, well illustrated in the definition provided by the Royal College of Physicians as "an active process of change by which a person who has become disabled acquires and uses the knowledge and skills necessary for optimal physical, psychological and social function". So although we may be able to identify certain key components such as education and self management, the ways in which these aims are achieved may vary greatly and are not easy to standardise. There are also other difficulties, including an inbuilt reluctance to use a control group, inadequate or absence of blinding, and a failure to use independent assessors.

There is some encouragement to be taken from the stroke literature, again bedeviled by many relatively small and methodologically limited studies, most of which suggested that coordinated stroke care or stroke units improve outcome but none of which was powerful enough to show it conclusively. It was only when a meta-analysis was carried out under the auspices of the Cochrane Collaboration that the message became irrefutable-stroke units reduce mortality and morbidity and increase the likelihood of discharge to home for patients with mild, moderate, and severe stroke. ${ }^{41}$

Stroke is, of course, a so called single incident pathology (though up to $50 \%$ of those that survive are left with considerable disability) and additional difficulties are encountered when addressing progressive neurological disorders such as multiple sclerosis. Indeed, there are those who think that rehabilitation is inappropriate for progressive disorders, displaying a lamentable ignorance of its underlying philosophy. ${ }^{42}$ Multiple sclerosis is one of the major causes of neurological disability and has a devastating effect on the young adult population which it attacks - affecting employment, relationships, and social role and costing, in this country, in excess of $£ 1.2$ billion a year. However, this sceptical view does place additional pressure to demonstrate both the appropriate- 
ness and effectiveness of rehabilitation in this area. ${ }^{43}$ This evaluation may be carried out at four different levels, moving from (1) the broad concept of comprehensive service delivery, through (2) packages of rehabilitation based on inpatient, outpatient, or community setting, (3) components of the package such as physiotherapy, and finally the most challenging area (4) the intrinsic elements of the rehabilitation process such as assessment and selection-sometimes referred to as the "black box" of rehabilitation and to date left largely undisturbed. Inpatient rehabilitation is perhaps more easily evaluated and has been the subject of some recent studies. ${ }^{44}$ The two key questions to be addressed are: (1) Is comprehensive inpatient rehabilitation effective in reducing disability and handicap and improving quality of life? (2) How long do benefits carry over for in this progressive neurological disorder?

As with stroke, some small single group studies, limited to impairment and disability, have suggested that rehabilitation does result in some benefit in multiple sclerosis which persists, at least in the short term. More recently, a study was designed which attempted to address some of the methodological issues mentioned earlier. ${ }^{45}$ It was randomised and the reluctance to have a control group was overcome by having wait list controls; in other words, every control patient received rehabilitation after a short wait - in this case six weeks. (Compared with current NHS waiting times, this could be seen as a positive benefit!) Patients were also stratified for disability to ensure that the two arms were well balanced. The assessor was blinded although of course the patient was not. The number in the study was small (66 patients with progressive multiple sclerosis) but none the less a significant benefit in disability measured with the Functional Independence Measure $(p<0.001)$ and handicap measured with the London Handicap Scale $(\mathrm{p}<0.01)$ was seen, despite unchanging disease status (EDSS).

The important, but difficult, area of carry over after rehabilitation input has also been addressed recently by Freeman et al in a single group longitudinal study of 50 patients with progressive multiple sclerosis. ${ }^{46}$ This study used a broader range of measures incorporating quality of life and emotional wellbeing (measured by the General Health Questionnaire) and demonstrated that despite the progressive nature of the condition, benefits were maintained after discharge in all areas. However, the time span, which was conservatively estimated using summary measures, varied. In disability (perhaps the most straightforward) it was maximum on discharge and worsened slowly returning to baseline by about 6 months. Handicap, on the other hand and somewhat predictably, continued to improve after discharge before slowly returning to baseline, indicating the important role of the home environment. Perhaps most strikingly, the mental component of the SF-36 and emotional wellbeing did not lose their benefit, possibly reflecting the educational and "coping" focus of rehabilitation. These data indicate that each of the measures used is addressing a different but complementary area and suggests that it might be appropriate to have a range of measures when attempting to evaluate rehabilitation. This study was widely commented on, again emphasising the increasing awareness of this area-indeed it even reached the dizzy heights of the Italian equivalent of Time, appropriately named L'espresso. (Its impact factor has yet to be determined but it is certainly very influential among the coffee drinking population of Italy.)

Two major points arise from these and other recent studies. ${ }^{47}$ Firstly, that it is now possible to carry out rigorous, scientific evaluation of rehabilitation, and these studies are "a few steps in the right direction" 48 although perhaps not quite as encouraging as suggested recently by Kraft. ${ }^{49}$ Secondly, they underline the need for a continuity between hospital and community with an emphasis on community care. This has been the major stimulus for change in health care delivery over the past decade and which one might have hoped would have produced results by now. How disappointing then to read the title of a recent detailed survey carried out by the Stroke Association "Stroke- still a matter of chance". ${ }^{50}$ The situation in multiple sclerosis is no different. In a recent study, the level of care provision in relation to disability was found to be erratic and inadequate. ${ }^{51}$ Perhaps of even greater concern, when the results were compared to those of McLellen et al, over 15 years ago, ${ }^{52}$ they were no better.

There remains much to be done in the management of neurological disability and, as discussed earlier, it will only be through basic science clarifying the mechanisms of disability and the application of scientifically sound outcome measures that interventions will make a real difference. To do this there has to be communication and collaboration between basic and clinical research and clinical practice. It places neuroscientists and clinical neurologists in a key position to exploit the ever increasing range of tools available to investigate not just mechanisms of disability but also mechanisms of recovery, such as neuroplasticity. An excellent example of such a tool is functional MRI (fMRI) which has already provided important insights into recovery in stroke but which is also being used tentatively in multiple sclerosis. ${ }^{53}$ Recently, a group of patients who had recovered from an episode of optic neuritis, often the first presentation of multiple sclerosis, were studied and a striking increase in brain activity outside the occipital cortex was seen when the previously affected eye was stimulated..$^{54}$ The areas activated were all known to have extensive visual connections (insulaclaustrum, lateral temporal and posterior parietal cortices, and thalamus) and it is postulated that they may play a part in the recovery process. Finally, it is possible to go one step further and combine structural techniques such as diffusion tensor imaging with $\mathrm{fMRI}^{55}$; clearly an area ripe for exploitation.

These developments have major implications for training in neurology which must now place 
a greater emphasis on the management of neurological disorders including stroke, head injury, and progressive conditions such as multiple sclerosis. It is encouraging to see that rehabilitation is now firmly incorporated into the neurology programme, a development facilitated by an appreciation of the need to incorporate rehabilitation services within neuroscience centres. Hopefully, these developments will result in the production of neurologists who can combine the philosophy of rehabilitation with an understanding of the basic neurosciences in a way which will improve our management of the many neurological disorders that cause such disability and distress to patients.

1 Lublin FD, Reingold SC, the National Multiple Sclerosis Society (USA) Advisory Committee on Clinical Trials of New Agents in Multiple Sclerosis. Guidelines for clinical trials of new therapeutic agents in multiple sclerosis. Relations between study investigators, advisors, and sponsors. Neurology 1997;48:572-4.

2 O'Riordan JI, Thompson AJ, Kingsley DPE, et al. The prognostic value of brain MRI in clinically isolated syndromes of the CNS. Brain 1998;121:495-503.

3 Miller DH, Grossman RI, Reingold SC, et al. The role of magnetic resonance techniques in understanding and manmagnetic resonance techniques in understanding

4 Thompson AJ, Miller DH, MacManus DG, et al. Patterns of disease activity in multiple sclerosis. BMF 1990;301:44-5.

5 Thompson AJ, Kermode AG, Wicks D, et al. Major differences in the dynamics of primary and secondary progressive multiple sclerosis. Ann Neurol 1991;29:53-62.

6 Kidd D, Thorpe JW, Thompson AJ, et al. Spinal cord MRI using multi-array coils and fast spin echo. II. Findings in multiple sclerosis. Neurology 1993;43:2632-7.

7 Kidd D, Thorpe JW, Kendall BE, et al. MRI dynamics of brain and spinal cord in progressive multiple sclerosis. $f$ Neurol Neurosurg Psychiatry 1996;60:15-19.

8 Losseff NA, Webb SL, O'Riordan JI, et al. Spinal cord atrophy and disability in multiple sclerosis. A new reproducible and sensitive MRI method with potential

9 Stevenson VL, Leary SM, Losseff NA, et al. Spinal cord atrophy and disability in MS. A longitudinal study. Neurology 1998;51:234-8.

10 Losseff NA, Wang L, Lai HM, et al. Progressive cerebral atrophy in multiple sclerosis. A serial study. Brain atrophy in multip

11 Simon JH, Jacobs LD, Campion MK, et al. A longitudinal study of brain atrophy in relapsing multiple sclerosis. $\mathrm{Neu}$ rology 1999;53:139-48.

12 Fox NC, Jenkins R, Leary SM, et al. Progressive cerebral atrophy in MS. A serial study using registered, volumetric MRI. Neurology 2000;54:807-12.

13 Jagust WJ, Noseworthy JH. Brain atrophy as a surrogate marker in MS. Faster, simpler, better? Neurology 2000;54: $782-3$.

14 Ferguson B, Matyszak MK, Esiri MM, et al. Axonal damage in acute multiple sclerosis lesions. Brain 1997;120:393-9.

15 Trapp BD, Peterson J, Ransohoff RM, et al. Axonal transaction in the lesions of multiple sclerosis. N Engl f Med1998; 338: $278-85$.

16 Revesz T. Axonal lesions in multiple sclerosis: an old story revisited. Brain 2000;123:203-4.

17 Allen IV, Glover G, Anderson R. Abnormalities in the macroscopically normal white matter in cases of mild or spinal multiple sclerosis (MS). Acta Neurophathol 1981; VII(supp(Berl)): 176-78.

18 Filippi M, Rocca MA, Martino G, et al. Magnetization transfer changes in the normal appearing white matter precede the appearance of enhancing lesions in patients with multiple sclerosis. Ann Neurol 1998;43:809-14.

19 Werring DJ, Clark CA, Barker GJ, et al. Diffusion tensor imaging of lesions and normal appearing white matter in MS. Neurology 1999;52:1626-32.

20 Davie CA, Barker GJ, Webb S, et al. Persistent functional deficit in multiple sclerosis and autosomal dominant cerebellar ataxia is associated with axon loss. Brain 1995;118:1583-92.

$21 \mathrm{Fu} \mathrm{L}$, Matthews PM, De Stefano N, et al. Imaging axona damage of normal-appearing white matter in multiple sclerosis. Brain 1998;121:103-13.

22 De Stefano N, Narayanan S, Matthews PM, et al. In vivo evidence for axonal dysfunction remote from focal cerebral demyelination of the type seen in multiple sclerosis. Brain 1999;122:1933-9.

23 van Waesberghe JHTM, Kamphorst W, De Groot CJA, et al. Axonal loss in MS lesions: MR insights into substrates of disability. Ann Neurol 1999;46:747-54

24 Kurtzke JF. Rating neurological impairment in multiple sclerosis: an expanded disability status scale (EDSS). Neurology 1983;33:1444-52
25 Guilford JP. Psychometric methods. New York: McGraw-Hill, 1936.

26 Hobart JC, Lamping DL, Thompson AJ. Evaluating neurological outcome measures: the bare essentials [editorial]. $\mathcal{F}$ Neurol Neurosurg Psychiatry 1996;60:127-30.

27 Freeman JA, Hobart JC, Langdon DW, et al. Clinical appropriateness: a key factor in outcome measure selection: the 36 item short form health survey in multiple sclerosis. F Neurol Neurosurg Psychiatry 2000;68:150-6.

28 World Health Organization. International classification of impairments, disabilities and handicaps. Geneva: WHO, 980:449-508.

29 Mahoney FI, Barthel DW. Functional evaluation: the Barthel index (BI). Maryland State Medical fournal 1965;14:61-5

30 Granger CV, Cotter AC, Hamilton BB, et al. Functional assessment scales: a study of persons with multiple sclerosis. Arch Phys Med Rehabil 1990;71:870-5.

31 Harwood RH, Rogers A, Dickinson E, et al. Measuring handicap. London handicap scale, a new outcome measure for chronic disease. Quality in Health Care 1994;3:11-16.

32 Rothwell PM, McDowell Z, Wong CK, et al. Doctors and patients don't agree: cross sectional study of patients' and doctors' perceptions and assessments of disability in multiple sclerosis. BMf 1997;314:1580-3.

33 Hobart JC, Freeman JA, Lamping DL. Physician and patient-oriented outcomes in progressive neurological disease: which to measure? Curr Opin Neurol 1996;9:441-

34 Vickrey BG. Getting oriented to patient-oriented outcomes. Neurology 1999;53:662-3.

35 Ware JE, Sherbourne CD. The MOS 36-item short form health survey $(\mathrm{SF}-36)$. 1. Conceptual framework and item selection. Med Care 1992;30:473-83.

36 Hobart J, Freeman JA, Thompson AJ. Kurtzke scales revisited: the application of psychometric methods to clinical intuition. Brain 2000;123:1027-40.

37 Van der Putten JJMF, Hobart JC, Freeman JA, et al. Measuring change in disability after inpatient rehabilitation: comparison of the responsiveness of the Barthel Index and functional independence measure. F Neurol Neurosurg Psychiatry 1999;66:480-4.

38 Hobart J, Lamping D, Freeman J, et al. Measuring neurology: Is bigger better? Comparative measurement properties of the functional independence measure (FIM) and Barthel index. Neurology 1997;48:A235.

39 Hobart JC, van der Putten JJ, Freeman JA, et al. Development and testing of a short form Barthel Index. $\mathcal{F}$ Neurol Neurosurg Psychiatry 1999;67:264.

40 Hobart JC, Lamping DL, Fitzpatrick R, et al. Patient based outcome measures for multiple sclerosis are needed and can be developed. $\mathcal{F}$ Neurol Neurosurg Psychiatry 2000;69: 000-000 (in press).

41 Langhorne P, Dennis M. eds. Stroke units: an evidence based approach. London: BMJ, 1998.

42 Thompson AJ. Rehabilitation of progressive neurological disorders: a worthwhile challenge. Curr Opin Neurol 1996; 9:437-40.

43 Thompson AJ. The effectiveness of neurological rehabilitation in multiple sclerosis. F Rehabil Res Dev 2000 (in press).

44 Freeman JA, Thompson AJ. Inpatient rehabilitation in multiple sclerosis. International MS fournal 1998;5:16-23.

45 Freeman JA, Langdon DW, Hobart JC, et al. The impact of inpatient rehabilitation on progressive multiple sclerosis. Ann Neurol 1997;42:236-44.

46 Freeman JA, Langdon DW, Hobart JC, et al. Inpatient rehabilitation in multiple sclerosis: do the benefits carry over into the community? Neurology 1999;52:50-6.

47 Solari A, Filippini G, Gasco P, et al. Physical rehabilitation has a positive effect on disability in multiple sclerosis patients. Neurology 1999;52:57-62.

48 Aisen ML. Justifying neurorehabilitation: a few steps forward. Neurology 1999;52:8-10

49 Kraft GH. Rehabilitation still the only way to improve function in multiple sclerosis. Lancet 1999;354:2016-17.

50 Stroke care. A matter of chance: a national survey of stroke services. London: The Stroke Association, 1999.

51 Freeman JA, Thompson AJ. Community services in multiple sclerosis: still a matter of chance. $\mathcal{F}$ Neurol Neurosurg Psychiatry 2000;69:728-32.

52 McLellan DL, Martin JR, Roberts MHW, et al. Multiple sclerosis in the Southampton district. Southampton: University of Southampton: Rehabilitation Research Unit and Department of Sociology and Social Policy, 1989. (Technical report 2. ISNB 085432318X.)

53 Lee M, Reddy H, Johansen-Berg H, et al. The motor cortex shows adaptive functional changes to brain injury from multiple sclerosis. Ann Neurol 2000;47:606-13.

54 Werring DJ, Bullmore ET, Toosy AT, et al. Recovery from optic neuritis is associated with a change in the distribution of cerebral response to visual stimulation: a functional magnetic resonance imaging study. 7 Neurol Neurosurg Psychiatry 2000;68:441-9.

55 Werring DJ, Clark CA, Barker GJ, et al. The structural and functional mechanisms of motor recovery: complementary use of diffusion tensor and functional magnetic resonance imaging in a traumatic injury of the internal capsule. $\mathcal{F}$ Neurol Neurosurg Psychiatry 1998;65:863-9. 